\title{
Improving Fingerprint Matching Using an Orientation-Based Minutia Descriptor
}

\author{
Miguel Angel Medina-Pérez, Andrés Gutiérrez-Rodríguez, \\ and Milton García-Borroto \\ Centro de Bioplantas, C. de Ávila, Cuba \\ \{migue, andres, mil\} @bioplantas.cu \\ http://www.bioplantas.cu
}

\begin{abstract}
This paper reviews a well-known fingerprint matching algorithm that uses an orientation-based minutia descriptor. It introduces a set of improvements to the algorithm that increase the accuracy and speed, using the same features. The most significant improvement is in the global minutiae matching step, reducing the number of local matching minutiae and using multiple minutiae pairs for fingerprint alignment. We conduct a series of experiments over the four databases of FVC2004, showing that the modified algorithm outperforms its predecessor and other algorithms proposed in the literature.
\end{abstract}

Keywords: biometrics, fingerprint matching, orientation-based minutia descriptor.

\section{Introduction}

Fingerprint recognition [1] has become one of the most active research areas nowadays. It plays an important role in forensic applications, but its increasing popularity is perhaps due to its integration into civilian systems. A key point in most of its applications is the fingerprint matching algorithm.

Most of the authors distinguish two types of fingerprint matching algorithms: correlation-based matching and minutiae-based matching. As it is seen in the Fingerprint Verification Competitions (FVC) [2], the minutia-based matching is the most popular approach. This approach essentially consists on finding the maximum number of matching minutiae pairs given two fingerprints represented by their minutiae.

Minutiae are the points where the ridge continuity breaks and it is typically represented as a triplet $(x, y, \theta)$; where $x$ and $y$ represents the point coordinates and $\theta$, the ridge direction at that point. As pointed out by Feng in [3], this representation makes ambiguous the process of minutia pairing. A way to deal with this problem is enriching the minutia representation with additional information known as minutia descriptors. Minutia descriptors can be mainly classified in: ridge based descriptors [4-8], orientation based descriptors [4, 9-11] and local neighboring minutiae based descriptors [3, 12-14].

This paper reviews the algorithm created by Tico and Kuosmanen [11] (TK hereafter), and proposes improvements in the matching algorithm using the same minutia descriptor. The new proposal increases the accuracy in terms of ERR, ZeroFMR, 
1000FMR and 100FMR. It also reduces the matching time according to the evaluation protocols of FVC [2].

We have structured the rest of the paper as follows. Section 2 describes TK algorithm. Section 3 describes the new formulation and improvements of TK and provides some details on the implementation of the new algorithm. Section 4 details the experimental results over FVC2004. Finally, the paper provides conclusions about the new algorithm that might be the starting point for new researches.

\section{TK Algorithm}

TK compares fingerprints by means of local minutiae structures, also known as minutiae descriptors. Minutiae descriptors provide additional information that enriches the minutia; and they are usually invariant to rotation and translation. TK uses a descriptor based on the estimations of the orientation values on sampling points that have been arranged in concentric circles around the minutia (see Fig. 1).

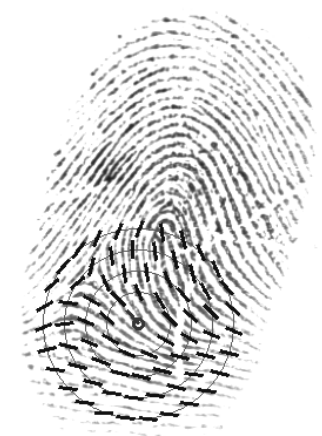

Fig. 1. A representation of the minutia descriptor proposed in [11]

Let $L$ be the amount of circles with $K_{l}$ sampling point each; given a minutia $\boldsymbol{q}=(x, y, \theta)$ we express its associated descriptor as:

$$
e(\boldsymbol{q})=\left(\left(\delta_{l, k}\right)_{k=1}^{K_{l}}\right)_{l=1}^{L} .
$$

Where $\delta_{l, k}$ is the angle difference between the minutia direction $\theta \in[0,2 \pi[$ and the fingerprint orientation value $\vartheta_{l, k} \in\left[0, \pi\right.$ [ in the $k^{t h}$ point of the $l^{t h}$ circle (the reader can refer to [1] for the conceptual difference between direction and orientation). We compute the angle difference as:

$$
\begin{gathered}
\delta_{l, k}=\min \left\{d\left(\theta, \vartheta_{l, k}\right), d\left(\theta, \vartheta_{l, k}+\pi\right)\right\}, \\
d(\alpha, \beta)=\min \{|\alpha-\beta|, 2 \pi-|\alpha-\beta|\} .
\end{gathered}
$$

Equation (2) computes the minimum angle required to make two lines parallel, if they have angles $\theta$ and $\vartheta_{l, k}$ respectively. 
TK consist of two major steps: local minutiae matching and global minutiae matching. In the local minutiae matching step, for each query minutia $\boldsymbol{q}_{j} \in Q=$ $\left\{\boldsymbol{q}_{1}, \boldsymbol{q}_{2}, \ldots, \boldsymbol{q}_{n}\right\}$ and for each template minutia $\boldsymbol{p}_{i} \in T=\left\{\boldsymbol{p}_{1}, \boldsymbol{p}_{2}, \ldots, \boldsymbol{p}_{m}\right\}$, the algorithm computes the possibility value as follows:

$$
P\left(\boldsymbol{q}_{j}, \boldsymbol{p}_{i}\right)=\frac{s\left(\boldsymbol{q}_{j}, \boldsymbol{p}_{i}\right)^{2}}{\sum_{\substack{h=1 \\ h \neq j}}^{n} s\left(\boldsymbol{q}_{h}, \boldsymbol{p}_{i}\right)+\sum_{\substack{h=1 \\ h \neq i}}^{m} s\left(\boldsymbol{q}_{j}, \boldsymbol{p}_{h}\right)-s\left(\boldsymbol{q}_{j}, \boldsymbol{p}_{i}\right)} .
$$

This expression returns high values when the similarity value $s\left(\boldsymbol{q}_{j}, \boldsymbol{p}_{i}\right)$ is large, and minutiae $\boldsymbol{q}_{j}$ and $\boldsymbol{p}_{i}$ have small similarities with respect to the other minutiae from $Q \backslash\left\{\boldsymbol{q}_{j}\right\}$ and $T \backslash\left\{\boldsymbol{p}_{i}\right\}$ respectively. Let $K=\sum_{l=1}^{L} K_{l}$, given the minutiae $\boldsymbol{q}_{j}$ and $\boldsymbol{p}_{i}$, TK computes their similarity as:

$$
s\left(\boldsymbol{q}_{j}, \boldsymbol{p}_{i}\right)=1 / K \sum_{l=1}^{L} \sum_{k=1}^{K_{l}} \exp \left(-16(2 / \pi)\left(\left|\delta_{l, k}^{j}-\delta_{l, k}^{i}\right|\right)\right) .
$$

In the global minutiae matching step, TK sort all minutiae pairs in descendent order, according to their possibility value. It transforms the query minutiae according to the minutiae pair that maximizes the possibility value. Then, it uses a greedy algorithm to find the minutiae pairs that satisfy the following constraints:

- The Euclidean distance between the two minutiae does not exceed threshold $t_{s}$.

- The difference between the two minutiae directions does not exceed threshold $t_{\theta}$.

Finally, TK uses the global matching minutiae, together with those minutiae that fall inside the region of interest that is common to both fingerprints, to compute the matching score. The minutiae count inside the region of interest common to both fingerprints must exceed threshold $t_{m}$.

The parameters of the algorithm are: distance threshold $t_{s}$, angle threshold $t_{\theta}$ and minutia count threshold $t_{m}$.

The next section analyzes some of the drawbacks of this algorithm and proposes modifications to overcome these limitations.

\section{The Modified TK Algorithm}

TK algorithm only uses the local matching minutiae pair that maximizes the possibility value to align fingerprints in the global minutiae matching step. The fact that a minutiae pair maximizes the possibility value does not guarantee that this is a true matching minutiae pair (see Fig. 2). Moreover, if the selected minutiae pair was a true matching pair, it is not necessarily the best pair to carry out fingerprint alignment.

In order to deal with this limitation, the new algorithm first reduces the local matching minutiae pairs by selecting, for each query minutia $\boldsymbol{q}_{j}$ and template minutia $\boldsymbol{p}_{i}$, only the minutiae pair $\left(\boldsymbol{q}_{j}, \boldsymbol{p}_{i}\right)$ that maximizes the possibility value. Then it 


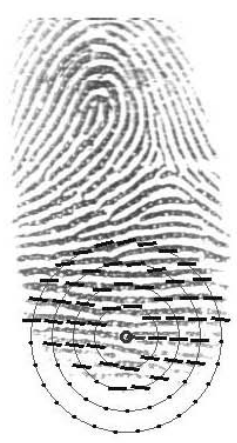

(a)

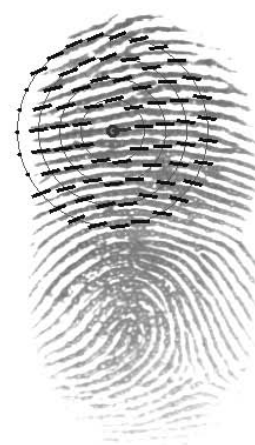

(b)

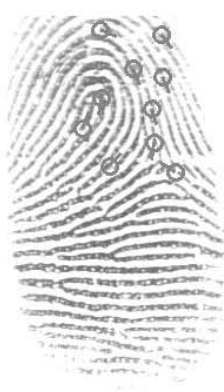

(c)

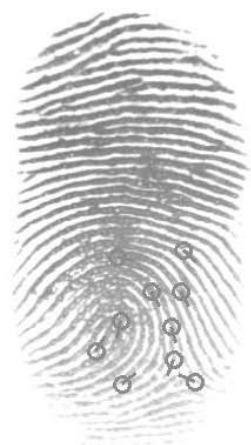

(d)

Fig. 2. The matching minutiae found by TK in fingerprints (a) 11_1 and (b) 11_3 from DB1_A in FVC2004. This is an example of a false matching minutiae pair that maximizes the possibility value in two fingerprints from the same finger. Images (c) and (d) show the good behavior of the modified TK algorithm while the original TK fails.

performs a query minutiae transformation for each minutiae pair in the reduced set. Finally, it selects the transformation that maximizes the amount of global matching minutiae pairs. This modification, like the global minutiae matching step of TK, has quadratic time complexity with respect to fingerprint minutiae count.

Another weakness of TK algorithm is that it does not take advantage of the small image rotation on the fingerprint verification problems. Therefore, we propose a modification of equation (5), which increases the minutia discrimination for such problems. We define the new minutia similarity as follows:

$$
s\left(\boldsymbol{q}_{j}, \boldsymbol{p}_{i}\right)=\left\{\begin{array}{cc}
0 & \text { if } d\left(\theta_{j}, \theta_{i}\right)>\frac{\pi}{4} \\
1 / K \sum_{l=1}^{L} \sum_{k=1}^{K_{l}} \exp \left(-16(2 / \pi)\left(\left|\delta_{l, k}^{j}-\delta_{l, k}^{i}\right|\right)\right) & \text { otherwise }
\end{array} .\right.
$$

The last modification that we propose is to limit the minimum count of global matching minutiae instead of bounding the minimum minutiae count inside the region of interest that is common to both fingerprints. We introduce this modification based on the forensic criterion that a true matching fingerprints pair must have at least $t_{m}$ true matching minutiae pairs [1] $\left(t_{m}\right.$ varies for different countries).

We name the new formulation of TK as Modified TK algorithm (MTK). A formal description of MTK is the following:

1. Let $T=\left\{\boldsymbol{p}_{1}, \boldsymbol{p}_{2}, \ldots, \boldsymbol{p}_{m}\right\}$ and $Q=\left\{\boldsymbol{q}_{1}, \boldsymbol{q}_{2}, \ldots, \boldsymbol{q}_{n}\right\}$ be the template and query fingerprint minutiae set respectively. For each query minutia $\boldsymbol{q}_{j} \in Q$ and for each template minutia $\boldsymbol{p}_{i} \in T$, compute the possibility value using equation (4).

2. Sort in descendent order all pairs $\left(\boldsymbol{q}_{j}, \boldsymbol{p}_{i}\right)$ according to the possibility value and store in $R \leftarrow\left\{\left(\boldsymbol{q}_{j, 1}, \boldsymbol{p}_{i, 1}\right),\left(\boldsymbol{q}_{j, 2}, \boldsymbol{p}_{i, 2}\right), \ldots,\left(\boldsymbol{q}_{j, n m}, \boldsymbol{p}_{i, n m}\right)\right\}$.

3. Set $E \leftarrow\{\}$ and $R^{\prime} \leftarrow\{\}$. 
4. For each $\left(\boldsymbol{q}_{j, h}, \boldsymbol{p}_{i, h}\right) \in R, h=1, \ldots, n m$ do:

a. If $\boldsymbol{q}_{j, h} \notin E \vee \boldsymbol{p}_{i, h} \notin E$ then update $R^{\prime} \leftarrow R^{\prime} \cup\left\{\left(\boldsymbol{q}_{j, h}, \boldsymbol{p}_{i, h}\right)\right\}$ and $E \leftarrow E \cup$ $\left\{\boldsymbol{q}_{j, h}, \boldsymbol{p}_{i, h}\right\}$.

5. Set $C s \leftarrow\{\}$ and $Q s^{\prime} \leftarrow\{\}$.

6. For each $\left(\boldsymbol{q}_{j, h}, \boldsymbol{p}_{i, h}\right) \in R^{\prime}, h=1, \ldots, n$ do:

a. Set $E \leftarrow\{\}, C_{h} \leftarrow\{\}$ and $Q_{h}^{\prime} \leftarrow\{\}$.

b. For each $\left(\boldsymbol{q}_{j, g}, \boldsymbol{p}_{i, g}\right) \in R^{\prime}, g=1, \ldots, n$; if $\boldsymbol{q}_{j, g} \notin E \vee \boldsymbol{p}_{i, g} \notin E$ do:

i. Compute $\boldsymbol{q}_{j, g}^{\prime}=\left(x_{j, g}^{\prime}, y_{j, g}^{\prime}, \theta_{j, g}^{\prime}\right)$ as follows:

$$
\begin{aligned}
& {\left[\begin{array}{l}
x_{j, g}^{\prime} \\
y_{j, g}^{\prime} \\
\theta_{j, g}^{\prime}
\end{array}\right]=\left[\begin{array}{ccc}
\cos \Delta \theta & -\sin \Delta \theta & 0 \\
\sin \Delta \theta & \cos \Delta \theta & 0 \\
0 & 0 & 1
\end{array}\right]\left[\begin{array}{l}
x_{j, g}-x_{j, h} \\
y_{j, g}-y_{j, h} \\
\theta_{j, g}-\theta_{j, h}
\end{array}\right]+\left[\begin{array}{l}
x_{i, h} \\
y_{i, h} \\
\theta_{i, h}
\end{array}\right] \text { where } \boldsymbol{q}_{j, h}=} \\
& \left(x_{j, h}, y_{j, h}, \theta_{j, h}\right), \boldsymbol{p}_{i, h}=\left(x_{i, h}, y_{i, h}, \theta_{i, h}\right), \Delta \theta=\theta_{i, h}-\theta_{j, h}, \boldsymbol{q}_{j, g}= \\
& \left(x_{j, g}, y_{j, g}, \theta_{j, g}\right) .
\end{aligned}
$$

ii. Update $Q_{h}^{\prime} \leftarrow Q_{h}^{\prime} \cup\left\{\boldsymbol{q}_{j, g}^{\prime}\right\}$.

iii. Let $\boldsymbol{p}_{i, g}=\left(x_{i, g}, y_{i, g}, \theta_{i, g}\right)$; if $\sqrt[2]{\left(x_{j, g}^{\prime}-x_{i, g}\right)^{2}+\left(y_{j, g}^{\prime}-y_{i, g}\right)^{2}} \leq t_{s}$ and $d\left(\theta_{j, g}^{\prime}, \theta_{i, g}\right) \leq t_{\theta}$, then update $E \leftarrow E \cup\left\{\boldsymbol{q}_{j, g}, \boldsymbol{p}_{i, g}\right\}$ and $C_{h} \leftarrow$ $C_{h} \cup\left\{\left(\boldsymbol{q}_{j, g}, \boldsymbol{p}_{i, g}\right)\right\}$.

c. Update $C s \leftarrow C s \cup\left\{C_{h}\right\}$ and $Q s^{\prime} \leftarrow Q s^{\prime} \cup\left\{Q_{h}^{\prime}\right\}$

7. Select $C_{a} \in C s, Q_{a}^{\prime} \in Q s^{\prime}$ where $a=\operatorname{argmax}_{h=1, \ldots, n}\left|C_{h}\right|$.

8. If $\left|C_{a}\right|<t_{m}$ return 0 else: let $T_{Q^{\prime}}$ and $Q^{\prime}{ }_{T}$ represent the number of minutiae from $T$ and $Q_{a}^{\prime}$ respectively placed inside the intersection of the two fingerprint bounding rectangles, return $\frac{1}{T_{Q^{\prime} Q^{\prime}}}\left(\sum_{\left(\boldsymbol{q}_{j, h}, \boldsymbol{p}_{i, h}\right) \in C_{a}} s\left(\boldsymbol{q}_{j, h}, \boldsymbol{p}_{i, h}\right)\right)^{2}$.

Distance threshold $t_{s}$, angle threshold $t_{\theta}$ and minutia count threshold $t_{m}$ are parameters of the algorithm. The reader can refer to Fig. 2 to see the good behavior of the modified TK algorithm in a case where the original TK fails.

\section{Experimental Results}

In order to evaluate the new formulation of TK algorithm we make use of the four databases of FVC2004 and the performance evaluation protocols of this competition [2]. We express in percentage the performance indicators EER, FMR100, FMR1000 and ZeroFMR. The indicator Time refers to average matching time in milliseconds.

We use the same features and parameters for both TK and MTK algorithms in all databases: distance threshold $t_{s}=12$, angle threshold $t_{\theta}=\pi / 6$ and minutia count threshold $t_{m}=6$. We compute the features using the parameters that reported the best results in [11]. We carry out all the experiments on a laptop with an Intel Core Duo processor $(1.86 \mathrm{GHz})$ and $1 \mathrm{~GB}$ of RAM. 
Tables 1 to 4 show the experimental results of MTK compared to: the original TK, the minutiae matching algorithm proposed by Qi et al. [9] and the results reported by Het et al. [6]. The average matching time reported in [6] does not appear in these tables because the experiments were performed using a different computer hardware. We highlight with bold letter the best result for each performance indicator.

As we expected the algorithm MTK outperform TK in all the databases for each indicator. MTK is faster than TK because the modification that we introduce in equation (6) allows discarding several false matching minutiae without comparing the respective whole descriptors. Another important result is that MTK outperforms the rest of the algorithms in most of the databases.

MTK has some limitations despite the good results achieved in the experiments. The main factors that affect the good behavior of this algorithm are high fingerprint distortion and small fingerprint area (see Fig. 3).

Table 1. Experimental results on DB1_A of FVC2004

\begin{tabular}{llllll}
\hline Algorithm & EER(\%) & FMR100(\%) & FMR1000(\%) & ZeroFMR $(\%)$ & Time(ms) \\
\hline MTK & $\mathbf{7 . 6 3}$ & $\mathbf{1 5 . 8 2}$ & $\mathbf{2 2 . 0 7}$ & $\mathbf{2 8 . 5 4}$ & $\mathbf{7 . 3 7}$ \\
TK & 16.07 & 28.71 & 36.96 & 48.14 & 16.92 \\
Qi et al. [9] & 27.87 & 64.54 & 78.25 & 94.21 & 40.59 \\
He et al. [6] & 9.33 & 18.5 & 25.03 & 30.28 & - \\
\hline
\end{tabular}

Table 2. Experimental results on DB2_A of FVC2004

\begin{tabular}{llllll}
\hline Algorithm & EER(\%) & FMR100(\%) & FMR1000(\%) & ZeroFMR(\%) & Time(ms) \\
\hline MTK & $\mathbf{5 . 7 2}$ & $\mathbf{7 . 8 6}$ & $\mathbf{1 2 . 5 0}$ & $\mathbf{1 5 . 3 2}$ & $\mathbf{6 . 1 2}$ \\
TK & 8.45 & 13.43 & 20.25 & 26.18 & 13.77 \\
Qi et al. [9] & 28.49 & 59.32 & 68.64 & 90.64 & 31.53 \\
He et al. [6] & 7.34 & 13.39 & 16.6 & 19.89 & - \\
\hline
\end{tabular}

Table 3. Experimental results on DB3_A of FVC2004

\begin{tabular}{llllll}
\hline Algorithm & EER(\%) & FMR100(\%) & FMR1000(\%) & ZeroFMR $(\%)$ & Time $(\mathrm{ms})$ \\
\hline MTK & $\mathbf{3 . 7 7}$ & $\mathbf{7 . 3 6}$ & $\mathbf{1 3 . 6 4}$ & $\mathbf{2 0 . 6 8}$ & $\mathbf{1 0 . 9 8}$ \\
TK & 9.13 & 20.14 & 28.75 & 32.21 & 24.45 \\
Qi et al. [9] & 20.16 & 49.29 & 69.86 & 89.86 & 65.92 \\
He et al. [6] & 8.52 & 13.1 & 16.53 & 22.53 & - \\
\hline
\end{tabular}

Table 4. Experimental results on DB4_A of FVC2004

\begin{tabular}{llllll}
\hline Algorithm & EER(\%) & FMR100(\%) & FMR1000(\%) & ZeroFMR(\%) & Time(ms) \\
\hline MTK & 6.78 & 7.61 & 9.43 & 10.54 & $\mathbf{6 . 4 8}$ \\
TK & 7.72 & 11.21 & 18.07 & 43.25 & 14.18 \\
Qi et al. [9] & 26.15 & 60.29 & 70.32 & 82.25 & 33.09 \\
He et al. [6] & $\mathbf{2 . 7 1}$ & $\mathbf{4 . 2 1}$ & $\mathbf{5 . 5 7}$ & $\mathbf{7 . 0}$ & - \\
\hline
\end{tabular}


High fingerprint distortion causes minutia descriptors with distorted orientations values while small fingerprint area causes minutia descriptors with too many sampling points with no information at all; in both cases, the minutia descriptor matching is unreliable. Minutia descriptors based on local neighboring minutiae (see [12-14]) tends to be more robust to these problems. Therefore, MTK could be improved by two ways: enriching the minutia descriptor with local neighboring minutiae information or combining MTK with an algorithm based on local neighboring minutiae descriptor.

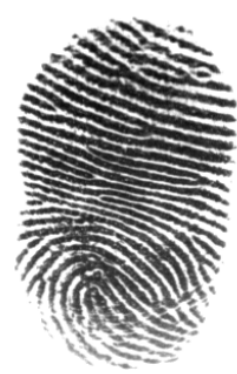

(a)

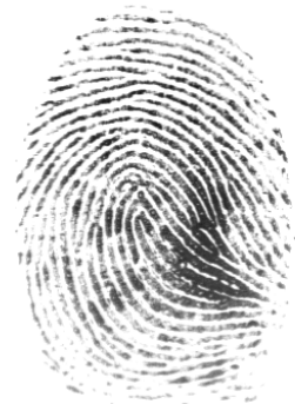

(b)

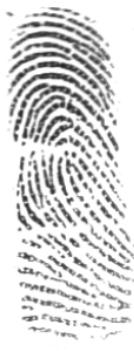

(c)

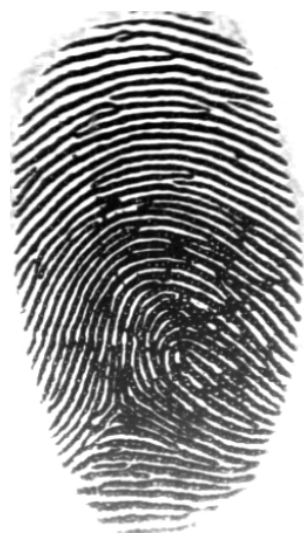

(d)

Fig. 3. These are two pairs of false not matching fingerprints from DB1_A using MTK. Fingerprints (a) 17_3 and (b) 17_6 are false not matching due to the high distortion on fingerprint (a). Fingerprints (c) 10_2 and (d) 10_4 are false not matching due to the small area of fingerprint (c).

\section{Conclusions}

This paper presents improvements to the fingerprint matching algorithm proposed by Tico and Kuosmanen in [11]. The new algorithm, named MTK, has three modifications of the original algorithm. First, we reduce the local matching minutiae pairs and use them all to accomplish a better fingerprint alignment. We introduce this modification because the minutiae pair that maximizes the possibility value is not necessarily a true matching minutiae pair; therefore, relying only on this pair for alignment may lead to false negative fingerprints matching. Second, we introduce a modification in the minutia similarity function in order to increase the minutiae discrimination with the additional advantage of reducing the matching time for fingerprint verification problems. Third, we include the forensic criterion that a true matching fingerprints pair must have at least certain count of true matching minutiae pairs. The conjunction of these modifications in MTK proves to be more accurate and faster than the original algorithm. The next step in our research is to investigate how the extensions of these modifications to other matching algorithms affect their performance. 


\section{References}

1. Maltoni, D., Maio, D., Jain, A.K., Prabhakar, S.: Handbook of Fingerprint Recognition. Springer, London (2009)

2. Cappelli, R., Maio, D., Maltoni, D., Wayman, J.L., Jain, A.K.: Performance evaluation of fingerprint verification systems. IEEE Trans. Pattern Anal. Mach. Intell. 28, 3-18 (2006)

3. Feng, J.: Combining minutiae descriptors for fingerprint matching. Pattern Recognit. 41, 342-352 (2008)

4. Wang, X., Li, J., Niu, Y.: Fingerprint matching using Orientation Codes and PolyLines. Pattern Recognit. 40, 3164-3177 (2007)

5. Feng, J., Ouyang, Z., Cai, A.: Fingerprint matching using ridges. Pattern Recognit. 39, 2131-2140 (2006)

6. He, Y., Tian, J., Li, L., Chen, H., Yang, X.: Fingerprint Matching Based on Global Comprehensive Similarity. IEEE Trans. Pattern Anal. Mach. Intell. 28, 850-862 (2006)

7. Luo, X., Tian, J., Wu, Y.: A minutiae matching algorithm in fingerprint verification. In: 15th International Conference on Pattern Recognition, Barcelona, Spain, vol. 4, pp. 833 836 (2000)

8. Jain, A.K., Lin, H., Bolle, R.: On-Line Fingerprint Verification. IEEE Trans. Pattern Anal. Mach. Intell. 19, 302-314 (1997)

9. Qi, J., Yang, S., Wang, Y.: Fingerprint matching combining the global orientation field with minutia. Pattern Recognit. Lett. 26, 2424-2430 (2005)

10. Tong, X., Huang, J., Tang, X., Shi, D.: Fingerprint minutiae matching using the adjacent feature vector. Pattern Recognit. Lett. 26, 1337-1345 (2005)

11. Tico, M., Kuosmanen, P.: Fingerprint matching using an orientation-based minutia descriptor. IEEE Trans. Pattern Anal. Mach. Intell. 25, 1009-1014 (2003)

12. Feng, Y., Feng, J., Chen, X., Song, Z.: A Novel Fingerprint Matching Scheme Based on Local Structure Compatibility. In: 18th International Conference on Pattern Recognition, Hong Kong, vol. 4, pp. 374-377 (2006)

13. Jiang, X., Yau, W.Y.: Fingerprint Minutiae Matching Based on the Local and Global Structures. In: 15th International Conference on Pattern Recognition, Barcelona, Spain, vol. 2, pp. 1038-1041 (2000)

14. Ratha, N.K., Bolle, R.M., Pandit, V.D., Vaish, V.: Robust fingerprint authentication using local structural similarity. In: Fifth IEEE Workshop on Applications of Computer Vision, Palm Springs, CA, USA, pp. 29-34 (2000) 